\title{
STUDIES ON THE ABSORPTION OF LIPIDS IN CALVES USING THE LYMPHATICO-VENOUS SHUNT TECHNIQUE
}

\author{
M. SIMPSON-MORGAN(1) and J. ZORRILLA-RIOS(2) \\ (1) Dept. Animal Production. University of Queensland. Australia \\ (2) Inst. Nac. Investigationes Pecuarias. Mexico
}

The direct quantitative determination of the apparent absorption of dietary fat in different species, including calves, has been reported to be measured by means of the cannulation of the intestinal or thoracic lymphatic ducts. This approach has also been used to study phenomena involved in the formation of lymph. A review of literature revealed contradictory lymphagogue characteristics of some nutrients, particularly fat, together with inconsistent values for the efficiency of absorption of diets of similar composition. Therefore, the validity of the lymphatico-venous shunt technique to determine the apparent absorption of dietary fat in liquid-fed calves was appraised.

Having found the traditional manual approach for collection, sampling and reinfusion of lymph a time-consuming, shortlasting, non-sterile technique, which is liable to cause a substantial degree of discomfort in the experimental animal and which is subject to great variation, we gradually improved it into an automatic system. The system which has finally been developed would seem to overcome most of the difficulties of lymph duct-blood vessel shunts and permits continuous monitoring of the flow and composition of lymph, with a minimal disturbance of the intraluminal hydrostatic pressure of the vessel, the aseptic state of the lymph or the physiological status of the animal. It allows the replacement of lymph almost as it is produced, so that it enters the blood almost as quickly as it would in the intact animal. The incorporation of an automatic fraction collector allows the monitoring of lymp to continue unattended on a $24 \mathrm{~h}$ a day basis. (see diagram).

A total of 31 calves were used and 70 experiments involving thirteen hour continous collections of lymph were done, during which the limitations of the technique for studies on lipid absorption and lymph formation in the intestines were delineated.

As part of these experiments, the automatic collection unit was used with fourteen calves. Termination of any preparation was usually due to accidents of one kind or another, such as pulling off of cannulae by the calves or electrical faults. For most of the experiments conduced under these conditions, the life of the shunts was 5-10 days. Certainly the number of experiments which could be done with individual calves using this automatic collection unit was very much greater than any described in literature. Flow was maintained in the last calf for 55 days, during which time the animal was weaned onto a solid diet.

The apparent digestibility of dietary fat was found to be unaffected by the surgical intervention necessary to establish the lymphatic fistulae, by the collection of lymph, or by the blockage of the intestinal lymphatic fistulae.

The results of an early series of experiments designed to provide information on the maximum capacity of the intestinal lymphatic 


\section{Pump and Line Assembly for Intestinal Lymph}

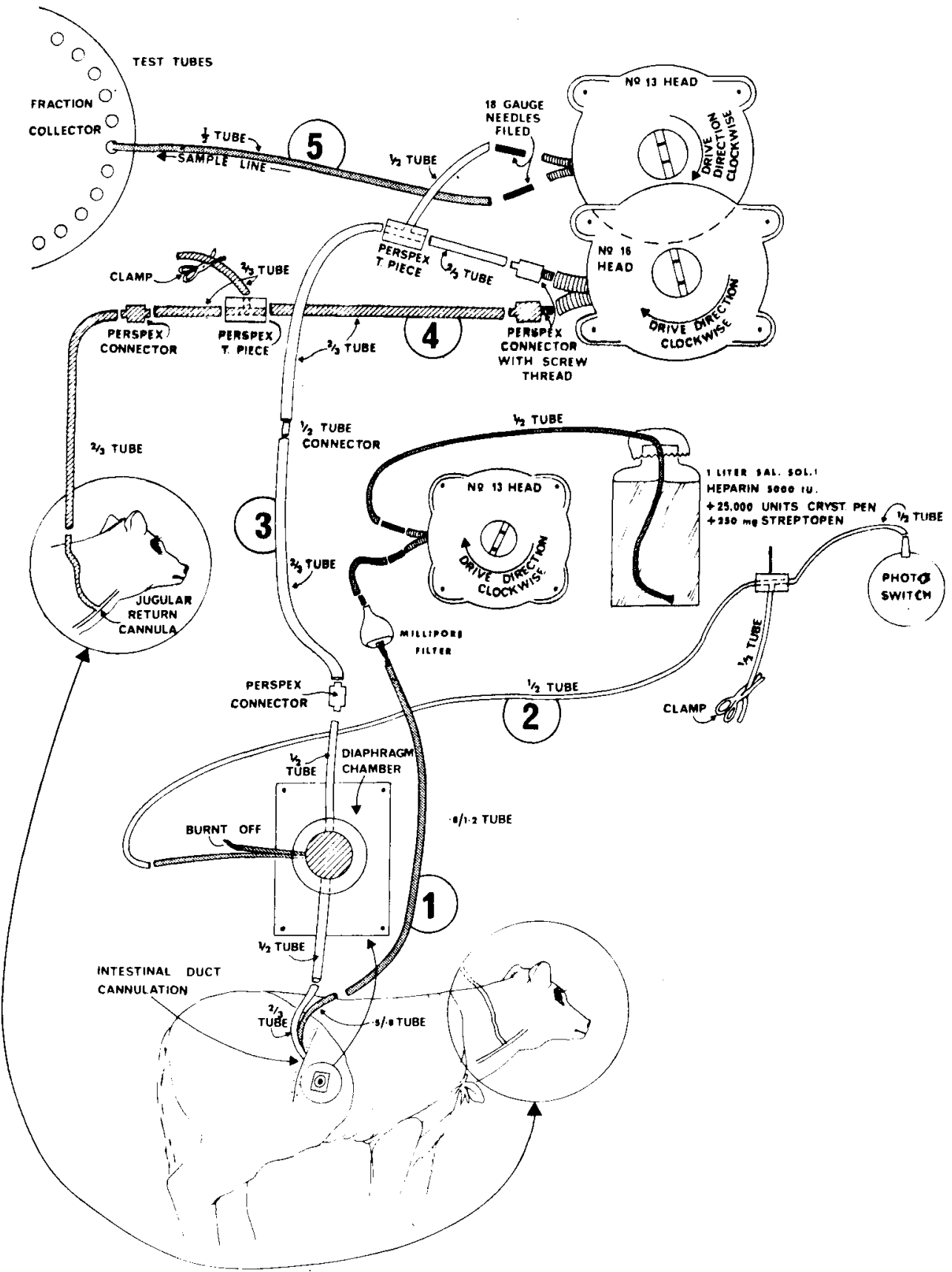

Diagrammatic representation of the automatic unit

1. Heparin line ; 2 . Line to operate the photo switch by transmiting changes in pressure within the diaphragm chamber; 3. Lymph collecting line ; 4 . Lymph recycling line ; 5 . Sampling line.

The figures on each tube indicate the internal and external diameters of the tube in millimetres. 
system of the milk-fed calf to transport fat revealed a limit of around $9 \mathrm{~g}$ of fat $/ \mathrm{h}$, during which the efficiency of absorption of dietary fat was of the order of $50 \%$. It was found that the different intakes of fat in the milk had no effect on the concentration of lipid in lymph and that the flow of lymph and the apparent absorption of dietary fat tended to decrease as the fat intake increased. As the pattern of transport of lipid in lymph reflected the pattern of abomasal emptying and passage of lipid from the abomasum into the intestine, a pattern previously determined for raw milk and skim milk plus tallow diets, a series of experiments was designed to determine if the previous results were a true limited capacity for the lymphatic absorption of lipid, or a reflection of a duodenal control on the emptying of the abomasum, in response to different amounts of lipid in the chyme. During these experiments, calves fed a fat-free diet (skim milk) were given continous infusions of cream directly into the duodenum, at different rates. Control collections for these studies were also compared with results from collection conducted after feeding a commercial milk replacer (Denkavit 2.5\% fat).

The main apparent results were : first, a limited capacity for the absorption of fat in lymph was once more apparent, this time over the range of $6-8 \mathrm{~g}$ of fat infusion $/ \mathrm{h}$. Second, the concentration of lipid in lymph reflected the intake of fat between the skim milk and the Denkavit treatment, but maintained similar levels after higher loads, both in the feed or during the duodenal infusion of cream. Third, an incomplete recovery of the duodenally infused fat in the intestinal lymph. Fourth, the absence of a lymphagogue effect during the absorption of fat in the flow of lymph when results from feeding skim milk or Denkavit were compared. Fifth, contrary to the lack of response of the concentration of lipid in lymph, the flow of lymph did respond to some rates of infusion. This response could not be reproduced by the infusion of the non-lipid fraction of the cream. Sixth, there was a delay of one to two hours between the initiation of the duodenal infusion of cream and its effect on the flow and composition of intestinal lymph.

In general, the transport of lipid in the lymph seemed much less than would be expected if all the digested dietary long chain fatty acids were trarisported in the lymph. In order to determine if the inconsistent recoveries of dietary fat in the intestinal lymph were due to incomplete collection of lymph flowing from the intestine, labelled fatty acids in the unesterified form or in the fatty acid of the triglyceride moiety were infused into the duodenum under a variety of conditions and their subsequent recovery in intestinal lymph monitored. Incomplete lymphatic recoveries of the labelled lipid infused into the duodenum suggested : a) the possibility of a by-pass of lymph at the point of cannulation by either lymphaticovenous anastomoses or actual branching of the intestinal duct not included in the original cannulation or, b) a direct transport of lipid by routes other than lymph, most likely the portal vein. None of these conditions could be demonstrated, therefore, it is possible that radioactive lipid which could not be accounted for in lymph or faeces was changed during passage through the gut by microbial activity into non-lipid substances which would not have been measured in radioassay of faecal lipids.

An effect of the carrier infused into the duodenum on the transit time of the chyme was recognized. This effect and the relationship between luminal conditions and the appearance of labelled lipid in lymph were further investigated using dual radioisotope infusions of lipids in the unesterified and esterified form, dissolved in either oleic acid or olive oil and infused into the duodenum at different times after feeding.

From these, it was concluded that the recovery of labelled lipid in intestinal lymph may reflect specific digestive processes taking place within the small intestine.

It is concluded that the study of absorption of lipids in lymph could contribute a great deal to an understanding of fat absorption in calves, by combining a number of the experimental techniques developed in these studies. These include the automatic continuous collection of lymph, adequate statistical design such as Latin Squares and multiple isotope methods, all of which allow many comparisons to be made in the same animal in a short period of time. The ability to replace radioactive lymph by reinfusing an adequate nonradioactive substitute at the same rate as the lymph is produced, overcomes many difficulties of recycling of labelled material in serial experiments. 